\title{
ARCHIV DER PHARMACIE.
}

3. Reihe, 1. Band, 4. Heft.

\section{A. Originalmitthellungen.}

\section{Chemie und Pharmacie.}

Ueber die Verwendung von Kaliselfe zur Bereltung des Selfenspiritus und fllissigen Opodeldocs.

Von G. H. Barakhaugen in Burgdorf bei Celle.

Es wird wohl jedem Fachgenossen bekannt sein, dass Seifenspiritus and fliussiger Opodeldoc, namentlich in der kalten Jahreszeit gelatiniren oder trübe werden durch Ausscheidung von weissen Seifenkörnchen. Man erwärmt oder filtrirt in solchen Fällen den Vorrath, und wenn man nach einigen Wochen wieder einfassen will, so findet man dieselbe Erscheinung; man erwärmt oder filtrirt wieder u. s. f.

Abgesehen davon, dass diese häufigen Erwärmungen oder Filtrationen Verluste an Zeit und Stoff verursachen, werden anch diese Präparate unrichtig. Diese Uebelstände haben ihren Grund darin, dass man zur Bereitung dieser Präparate Seifen verwendet, welohe mit Natron und wohl noch obendrein mit einem festen Fett bereitet sind.

Dies veranlasste mich schon im verflossenen Winter, zur Bereitung dieser Präparate Kaliseife in Gebrauch zu ziehen. Anfangs glaubte ich, dass die Schmierseife des Handels gerado hier am rechten Platzo sein uud einen nützlichen $Z_{\text {weck }}$ erfüllen würde, den ich, beiläufig bemerkt, in ihrer Verwendung im Haushalt and in der Technik nicht einsehen kann, wenn ich nur erwäge, dass man mit $47 \mathrm{Th}$. des theoren 
290 teber d. Verwendung v. Kaliseife z. Bereitung d. Seifenspiritus etc.

Aetzkali nicht mehr Fett verseifen kann, als mit $31 \mathrm{Th}$. des billigen Aetznatron, und ihre übrigen Eigenschaften, wie weiche Beschaffenheit, Gehalt an Glycerin und überschüssigem Alkali sich auch bei der Natronseife mit Leichtigkeit herstellen lassen. Was nun ihre Verwendung zu Seifenspiritus und flüssigem Opodeldoc betrifft, die mich mehr angeht, als die letzterwähnte, so hatte mir ein vorläufiger Versuch meine Voraussetzung soweit bestätigt, dass sich ein mit Schmierseife bereiteter Seifenspiritus selbst bei niedriger Temp. von 0 bis $+5^{\circ} \mathrm{C}$. klar hält ohne zu gelatiniren. Die Schmierseife kommt aber in so wechselnder Beschaffenheit im Handel vor, dass ich ihre Einfuihrung in den Arzneischatz selbst nicht zur Bereitung dieser äusserlichen Arzneimittel befuirworten möchte, denn abgesehen von dem oft sehr unangenehmen Geruch und der wechselnden Farbe enthält sie nicht selten noch andere erhebliche Verunreinigungen. Schon im Jahre 1868 theilt Roussin Bd. 136 S. 150 dieser Zeitschrift eine Verfälschung der Schmierseife mit, nach ihm enthielt alle schwarze Seife aus der Umgegend von Paris 20 bis $25 \%$ Stärke. Diese Verfälschung-kommt auch in Deutschland vor, ich behielt von einer hier am Platze gekauften Schmierseife beim Auflösen in Alkohol 17\% Stärke als Kleister im Filter zurück.

Somit bleibt kein anderer Ausweg, als sich die Kaliseife : für pharmaceutische Zwecke selbst $z \mathfrak{u}$ bereiten. Die erste Frage, die mir hierbei entgegentrat, war, wieviel wasserfreies reines Kali ist erforderlich, um $100 \mathrm{Th}$. Oel, ich wählte in diesem Falle Provenceröl, zu verseifen? Die Angaben, welche mir hierüber zur Verfügung sind und einen Schluss zulassen, insofern sie das Oel verseifen lassen, ohne auszusalzen, sind die Vorschrift der letzten Hannov. Pharmac. für Sapo medicatus und eine Mittheilung in diesem Archiv ron A. Vogel. Derselbe theilt daselbst Bd. 126, S. 151 mit, dass er durch Vermischen und schwaches Erwärmen von $20 \mathrm{Grm}$. koblensäurefreier Kalilauge von genau 1,33 sp. Gew., $40 \mathrm{Grm}$. Provenceröl, $120 \mathrm{Grm}$. Alkohol von 0,841 sp. Gew. und 120 c.c. Wasser in einem geräumigen Kolben sogleich eine ganz klare 
Ceber d. Verwendung $\vee$. Kaliseife z. Bereitung d. Seifenspiritus etc. 291

Lösung erhalten habe. Ich habe danach operirt und gefunden, dass man sehr lange, sogar bis zur vollständigen Entfernung des Alkohols erwärmen kann, obne eine Lösung des Oels zu erzielen; ein fortgesetztes Erhitzen lief darauf hinans, in der gewöhnlichen Weise einen Seifenleim darstellen zu wollen, und zwar mit einer zur Verseifung des Oeles nicht genügenden Menge Kalilange. Nach seiner Angabe waren nur 13,75 Th. $\mathrm{KO}$ in der Gestalt von $50 \mathrm{Th}$. Kalilauge von $1,33 \mathrm{sp}$. Gew. erforderlich, um $10 \mathrm{Th}$. Oel zu verseifen. Die frühere Hannov. Pharmac. lässt zur Bereitung der medicinischen Seife das Aetznatron aus 3 Th. Natr. carb. dep. bereiten und rerseift damit $3 \mathrm{Th}$. Provenceröl. Nimmt man an, dass das kohlensaure Natron chem. rein ist, und alles Aetznatron daraus gewonnen wird, so ergiebt das angewandte koblensaure Natron zur Verseifung von $100 \mathrm{Th}$. Provenceröl $21,67 \mathrm{Th}$. $\mathrm{NaO}$, woron allerdings wohl einige Verlustprocente in Abrechnung zu bringen sind. 21,67 Th. $\mathrm{NaO}$ sind äquivalent $32,85 \mathrm{Th}$. KO.

In der Voraussetzung, dass die richtige Menge KO zur Verseifung von 100 Th. Provenceröl zwischen diesen beiden Extremen liege, stellte ich eine Reihe von Versuchen an mit einer verdünnten Kalilauge, welche durch Kalkwasser nicht mehr gefällt wurde und nach dem Filtriren durch kohlensaures Kali auch keine Trübung mehr zeigte. Diese Kalilauge titrirte ich mit $1 / 10$ Normalsalzsänre, 1. c. c. $=0,00365 \mathrm{Grm}$. HCl. *)

- Anfange hatte ich mir eine Normalgäure aus der officinellen reinen Säure dargestellt, diese zeigte nach der Mohr'schen Waage bei $15^{\circ} \mathrm{C}$. ein sp. Gew. von 1,124 und enthielt demnach $25 \%$ HCl. $146 \mathrm{Grm}$. dieser Säure mit dest. Wasser zu 1 Liter verdünnt, enthalten demnach $36,5 \mathrm{Grm}$. HCl (das ist das Aeq. des Chlorwasserstoffs in Grammen ausgedrückt) oder 1 c. c. $=0,0365 \mathrm{Grm}$. $\mathrm{HCl}$. Angenommen, dass das sp. Gew. genau $25 \% \mathrm{HCl}$ anzeigt, so ist doch das Abwägen von $146 \mathrm{Grm}$. auf einex gewöhnlichen Waage nicht gensu genug, und es ist nöthig, die Normalsänre auf Silbernitrat einzustellen. Ich that dics auch, und fand, dass 1 Grm. AgONOs genau 6 c. c. Normalsäure zur Ausfällung des Ag erforderte. $\frac{36,5}{170}=0,2147$ und $\frac{0,2147}{0,0365}=5,88$. Ich hätte also statt 6 c.c. 
292 Ueber d. Verwendung v. Kaliseife z. Bereitung d. Scifenspiritus etc.

Bei der erwähnten Versuchsreihe gebrauchte ich zur Verseifung des Provenceröls von der verdünnten Kalilauge zunächst eine 28\% KO entsprechende Menge und ging dann in grossen Sprüngen zu 24 und $22 \%$ abwärts. Die erhaltenen Seifenleime waren spinnend und durchsichtig, gaben aber mit Wasser keine klare Lösung. Die Trübung konnte nicht herrühren von unverseiftem Oel, denn alle drei Proben gaben deutliche Reaction mit $\mathrm{HgCl}-$ Lösung, wie dies $\mathrm{W}$. Stein Bd. 148, S. 69 dieses Archivs als Prüfung auf freies Alkali empfohlen hat. Diese Erscheinung führte mich auf den Gedanken, dass diese Trübung von $\mathrm{Kalk}$ herrïhre und dass Kalilange selbst in verdiunntem Zustande nicht durch $\mathrm{KOCO}^{2}$ von $\mathrm{CaO}$ befreit werden könne. Fin Versuch mit reinem Kali bestätigte meine Vermuthung. Ich machte mir eine klare Lösung von Kali hydr. sicc. in Alkohol, verdampfte letzteren im Dampfbade und löste das zurïckbleibende reine

nur 5,88 c.c. verbraucht, wenn die Säure stark genug gewesen wäre, sie enthielt biernach also in 1000 c. c. nicht $36,5 \mathrm{Grm}$., sondern nur $35,77 \mathrm{Grm}$. HCl. Die Säure war also um $2 \%$ zu schwach und zeigte dessbalb $2^{\circ}$ o Alkali zu viel an. Hierbei ist mir bemerklich geworden, dass eine etwa vorkommende $\Delta$ blesung von 0,08 c.c. an einer in $1 / 5$ c.c. getheilten Bürette, wiê ich solche bisher gebrauchte, und wie sie noch häuflg benutzt wird, unmöglich wäre. Nun betragen abcr 0,08 c. c. bei einem Totalverbrauch von 6 c.c. eine Differenz von $1 \frac{1}{8} \%$. Um die gerühmte Ge. nauigkeit der Titrirmethode nicht zweifelhaft zu machen, scheint es mir gerathener, $1 / 10$ Normalflissigkeiten zu gebrauchen, und nur Büretten anzuwenden, die in $1 / 10$ c.c. getheilt, nnd bei einem Durchmesser von etwa $1 \mathrm{c.m}$. und einem Totalinhalt von 30 bis 35 c.c. nicht länger als 50 c.m. sind, damit man nicht nöthig hat, daran hinauf zu klettern, um das Auge mit dem Nivesu der Flüssigkeit in eine Horizontale zu bringen. Grosser Durchmesser und bedeutende Länge der Büretten sind Omstände, welche die Beobachtung erschweren. Wendet man Büretten von geringem Durchmesser und $1 / 10$ Normalfüssigkeiten an, und verbraucht man demnach zu einer Analyse eine grössere Anzahl c. c.', so ist es um so mehr nöthig, ror Ablesung etwa 1 Min. zu warten, damit die an den Wandungen der Bürette haftende Flüssigkeit herabfliesse. Di $\theta$ in dieser Arbeit angefihrten Analyeen habe ich mit einer in $1 / 10$ c.c. getheilten Bürette und $1 / 10$ Normalsalzsäure auggeführt, indem ich 102 c.c. der rermeintlichen Normalsäure mit destillirtem Wasser zu 1 Liter mischte. 
Deber d. Verwendung v. Kaliseife z. Bercitung d. Seifenspiritus etc, 293

Kali in dest. Wasser. Diese Lösung lässt sich in verschiedenen Concentrationsgraden mit einigen Tropfen Kalkwasser mischen, ohne sich zu trüben, und ein nachheriger Zusatz von $\mathrm{KOCO}^{2}$-Lösung fällt den $\mathrm{CaO}$ gar nicht oder nicht vollständig aus, versetzt man jetzt aber mit einer klaren Seifenlösung, so entsteht sofort ein Niederschlag ron Kalkseife. Eine alkoholische Lösung von Kalihydrat wird durch Seifenlösung nicht gefällt, ist also kalkfrei.

Ich stellte mir jetzt zum Zweck der Seifenbereitung eine grössere Menge alkoholischer Kalilösung dar, und bestimmte ihren Gehalt an KO mit $1 / 10$ Normalsalzeänre. Pelouze erwähnt in einer längeren Abhandlang über Verseifung der Oele, Journ. f. prakt. Chem. Bd. 65., dass dieselbe viel leichter von statten geht, wenn man eine innigere Mischung des Oels mit dem Alkali bewerkstelligt, und benutzt u. A. hierzu anch alkoholische Kali- und Natronlösungen. Der Verseifungsprocess vollzieht sich bei Anwendung der reinen Alkalien in alkoholischer Lösung in der That schnell, und wenn Pelonze sehr richtig bemerkt, dass diese Methode mit Nutzen bei akad. Vorlesungen angewendet werden könnte, und wenn man schon längst Alkohol zur Darstellung der transparenten Seifen fuir die Toilette anwendet, so dürfte sich ein derartiger Kostenaufwand mindestens ebenso sehr.empfehlen, wenn es sich um die Darstellung von Seifen für Heilzwecke handelt. Ich lasse hier eine Reihe von Versuchen folgen, welche über die Methode einigen Aufschluss geben.

Eine alkoholische Lösung von Kali oder Natron mischt sich bei $100^{\circ} \mathrm{C}$, fast in allen Verhältnissen mit fetten Oelen und Fetten zo einer klaren Flüssigkeit, die je nach der Nåtur des Alkalis und des Fettes und der Menge des angewandten Alkohols beim Erkalten flüssig bleibt oder erstarrt.

Erhitzt man Provenceröl mit je 15, 16, 16,5, 17, 18, 18,5 und $19,5 \% \mathrm{KO}$ in alkoholischer Lösung auf $100^{\circ} \mathrm{C}$, so erhält man klare Lösungen, welche alle mit $\mathrm{Hg} \mathrm{Cl}$-Lösung einen röthlichgelben oder rothen Niederschlag von $\mathrm{HgO}$ geben, also freies Alkali enthalten. Dieselben lassen sich 2war mit einer grossen Menge Wasser klar mischen, versetzt man jetzt 
aber mit wenig Alkohol, so trüben sich alle Lösungen mehr oder weniger, die mit 15 und $16 \% \mathrm{KO}$ scheiden sogar nach längerem Stehen dentlich $\mathrm{Oel} \mathrm{ab}$, obgleich sie zu gleicher Zeit ungebundenes Kali enthalten. Die alkalische Reaction verschwindet auch nicht, wenn man die alkoholischen Lösungen auf $125 \%$ des angewandten Oels verdampft, man erhält in diesem Falle Substanzen von dem Aussehen und der Consistenz der Wachssalbe, welche sich mit $\mathrm{HgCl}$-Lösung gelb bis roth färben.

Digerirt man die alkoholischen Lösungen stundenlang bei 65 bis $75^{\circ} \mathrm{C}$. unter Ersetzung des verdunsteten Alkohols, so findet man, dass das Oel je nach der Menge des angewandten, resp. verdunsteten Alkohols 15, 16 und 16,5\% KO chemisch bindet. Die alkalische Reaction der Proben mit 17 und $18 \%$ KO verschwand selbst nicht nach achtstündiger Digestion. Beim Verdampfen der so behandelten alkoholischen Lösungen auf $125 \%$ des angewandten Oels erhält man ebenfalls Substanzen von dem Aussehen und der Consistenz der Wachssalbe, setzt man diese Seifen tagelang der Luft aus, so ziehen sie Wasser an und bilden an ihrer Oberfläche eine durchsichtige schlüpfrige Gallerte. Hierbei beobachtete ich, dass sich die alkalische Reaction in demselben Grade verminderte. Ich versetzte hierauf die Probe der mit $18 \%$ KO bereiteten Seife mit wenig Wasser, mischte durch anhaltendes Rühren innig, und erhielt einen Seifenleim, der kein freies Alkali mehr enthielt. Was ich durch achtstündige Digestion mit Alkohol nicht erreichen konnte, erreichte ich in wenigen Minuten durch Mischen mit Wasser bei gewöhnlicher Temperatur.

Verdampft man dieselben alkoholischen Kali-Provenceröllösungen mit einer grossen Menge Wassers auf 300\% des angewandten Oeles, so erstarrt die Flüssigkeit vom Boden des Gefässes aus gallertartig und bildet beim Erkalten einen weniger oder mehr spinnenden Seifenleim. Hatte man viel Alkohol zur Lösung des Kalis verwendet, so zeigt der mit 18,5\% KO bereitete Seifenleim noch freies Alkali. Unter ähnlichen Umständen erhielt ich selbst mit $17 \%$ KO einen 
Ueber d. Verwendung v. Kaliseife z. Bureitung d. Seifeuspiritus etc. 295

Seifenleim, der noch freies Alkali enthielt. Bei anhaltender Digestion mit Wasser verschwindet die alkalische Reaction in beiden Fällen; wendet man aber 19,5\% $\mathrm{KO}$ an, so verschwindet die alkalische Reaction selbst nicht nach langer Digestion mit Wasser. Bei derselben Behandlung, d. h. bei Digestion mit Wasser und vollständiger Entfernung des Alkohols fand ich, dass Rüböl 16,5\% KO vollständig bindet, bei Anwendung von 17,5\% KO bleibt freies Alkali in dem Seifenleim selbst nach langer Digestion mit Wasser.

Da die Fettsäure des Oeles bei Gegenwart von Alkohol weniger KO bindet, als bei Gegenwart von Wasser, so combinirte ich weiter, dass der Alkohol auch eine fertige neutrale mit Wasser bereitete Seife zersetzen würde, und dies ist in der That der Fall. Ich löste $1 \mathrm{Th}$. mittelst Wasser mit $18,5 \%$ KO bereiteter Seife, welche an sich schon wasserhaltig ist, absichtlich zunächst in $1 \mathrm{Th}$. Wasser, um der Seife das zu ihrem Bestehen vermuthlich nöthige Wasser zu bieten. Die Seife in Substanz sowenig wie wässrige Lösung derselben gaben eine Reaction auf treies Kali, mischte ich aber die wässrige Lösung noch mit $1 \mathrm{Th}$. Alkohol, and prïfte dann mit $\mathrm{HgCl}$-Lösung, so entstand allmälig eine röthlichgelbe Truibung von $\mathrm{HgO}$. Eine Lösung derselben Seife in conc. Alkohol gab mit $\mathrm{HgCl}-\mathrm{Lösung}$ sofort einen rothen Niederschlag.

Ich konnte nun nicht unterlassen, von meinem Thema etwas abzuschweifen, um $z u$ erfahren, wie sich Natronseifen in alkoholischer Lösung verhalten. Ich löste stearinsaures Natron in $20 \mathrm{Th}$. Alkohol bei $70^{\circ} \mathrm{C}$, und versetzte mit $\mathrm{HgCl}$-Lösung bei einer Temp., bei welcher die Lösung noch eben flüssig war; es zeigte sich selbst in dieser verdünnten Lösung sofort ein rother Niederschlag, während die Stearinseife in Substanz sowenig wie in conc. wässriger Lösung eine Reaction auf freies Alkali zeigte.

Die vorherigen Versuche deuten darauf hin, dass der Alkohol die Zersetzung der Seife in freies Alkali und saures fettsaures Alkali durch Wasserentziehung bewirkt. Ein Gegenversuch, bei welchem ich die Entfernung des Wassers 
296 Deber d. Verwendung v. Kaliseife z. Bercitung d. Seijenspiritus ete.

durch Austrocknen bewirkte, macht dies noch wahrscheinlicher. Ich trocknete eine mit 18,5\% KO mittelst Wasser bereitete Provencerölseife bei $100^{\circ} \mathrm{C}$. anf etwa $140 \%$ des angewandten Oeles ein, und fand, dass dieselbe freies Alkali enthielt.

Die Thatsachen und Schlüsse, welche ich aus meinen Beobachtungen ziehe, und welche ich der Beachtung, Prüfung und weiteren Verfolgung empfehle, sind:

1) Kali- und Natronlauge können durch kohlensaures Alkali nicht von dem darin gelösten Kalk befreit werden, dies geschieht in allen Concentrationsgraden vollständiger durch Seifenlösung. Daher enthalten alle Seifen, welche mit einer Aetzlauge bereitet sind, deren Kalkgehalt nur durch kohlensaures Alkali ausgefállt war, Kalk. Die Lösung einer solchen Seife kann wohl klar erscheinen, wenn sie sich dem Concentrationsgrade eines Seifenleimes nähert, verdünnt man aber mit Wasser, so trübt sich die Lösung von sich ausscheidender Kalkseife.

2) Wenn man käufliches trocknes Aetzkali mit Alkohol auszieht, erhält man reines $\mathrm{Kali}$ in Lösung. Eine solche Lösung lässt sich bei $100^{\circ} \mathrm{C}$. fast in allen Verhältnissen mit Provenceröl klar mischen; diese Lösungen sind aber keine vollständig chemischen Verbindungen, in ein und derselben Lösung lässt sich freies Alkali neben unverseiftem $0 e l$ und der gebildeten Oelseife nachweisen.

3) Die Sättigungscapacität der Fettsäuren für KO und $\mathrm{NaO}$ bei Gegenwart von Alkohol ist geringer, als bei $\mathrm{Ge}$ genwart von Wasser, und es kann daher von einer Sättigungscapacität bei Gegenwart von Alkohol nur dann die Rede sein, wenn man absoluten Alkohol als Lösungsmittel anwendet. Bei [Anwendung von 90\% Alkohol variirt dieselbe merklich nach der Menge des zugesetzten, resp. bei mässiger Wärme mit der Seife verdunsteten Alkohols. (Sehr deutlich lässt sich dies nachweisen, wenn man 90\% Alkohol bei 30 bis $35^{\circ} \mathrm{C}$. mit der Seife digerirt, in diesem Falle bindet die Seife das Wasser aus dem Alkohol, während der Alkohol vorwiegend verdunstet). 
Uober d. Verwendung v. Kaliseife z. Bereitung d. Seifeuspiritus otc. 297

4) Um die Sättigungscapacität der Fetteäure für KO und $\mathrm{NaO}$ bei Gegenwart von Wasser, welche grösser ist, als die bei Gegenwart von Alkohol, zu erfahren, ist es umgekehrt nöthig, den Alkohol zu entfernen. Die in $100 \mathrm{Th}$. Provenceröl enthaltene Fettsäure bindet bei Gegenwart von Wasser 18,5 Th. KO und die in $100 \mathrm{Th}$. Rüböl enthaltene Fettsäure unter denselben Umständen 16,5 Th. KO.*) Bei Gegenwart von Alkohol binden diese Fettsäuren 2 bis 3 Th. KO weniger. Bei Darstellung der wässrigen Seifen hat der Alkohol den Zwock, ein reines Alkali darzustellen, und durch innige Berïhrung zwischen Fettsäure und Alkali den Verseifungsprocees zu beschleunigen.

5) Die mit Wasser bereiteten Seifen werden durch Alkohol in der Weise zersetzt, dass ein Theil des Alkalis frei wird und ein saures fettsaures Alkali entsteht. Der Grad dieser Zersetzung richtet sich nach der Stärke des Alkohols. Wir haben also im Seifenspiritus, sowie flüssigen und festen Opodeldoc freies Alkali neben saurem fettsauren Alkali, in Ijösung, welch letzteres eine der unvermeidlichen Ursachen der Trübung und Abscheidung von Sternchen im festen Opodeldoc sein kann.

6) Aehnlich, wie durch Alkohol, wird auch die mittelst Wasser mit 18,5\% KO bereitete Provencerölseife durch anhaltendes Trocknen bei $100^{\circ} \mathrm{C}$. zersetzt.

7) Die Sättigungscapacität der Fettsäuren für Kali und Natron lässt sich mit Hülfe titrirter Lösungen der reinen Alkalien und Prüfung mit $\mathrm{HgCl}$-Lösung genau verfolgen und bietet vielleicht eine brauchbare Prüfungsmethode auf Verfälschung fetter Oele.

*) Anmerkung. 18,5\% KO für Provenceröl und 16,5\% KO für Rüböl, sind nicht das Maximum, welches diese Oele binden, dasselbe mag sich vielleicht um $0,5 \%$ KO höher herausstellen; ich sehe aber keinen Grund ein, das Maximum oder gar einen Ueberschuss ron Alkali, wie bei der käuflichen Schmierseife der Fall ist, anzuwenden, da man mit den angegebenen Mengen KO vollkommen transparente, in Wasser und Alkohol sich klar lösende Seifen erhält. 
298 Ueber d. Verwendung v. Kaliseife z. Bcreitung d. Soifenspiritus etc.

Um aus den angefïhrten Thatsachen für die pharmaceutische Praxis zunächst zur Bereitung von Seifenspiritus und flüssigem Opodeldoc Nutzen zu ziehen, könnte man etwa, wie folgt verfahren. Um ganz sicher zu sein, dass man eine Seife erhält, deren Lösung in Alkohol selbst bei bedeutender Winterkälte nicht gelatinirt, wählt man $R$ üböl zur Verseifung. Man löst Kal. hydr. sicc. in möglichst wenig Alkohol, lässt absetzen und erwärmt im Dampfbade eine 16,5 Th. KO entsprechende Menge der Lösung mit $100 \mathrm{Th}$. Rüböl, bis letzteres gelöst ist. Durch fernere Digestion mit Wasser bis zur gänzlichen Entfernung des Alkohols und bis zum Verschwinden der alkalischen Reaction vollzieht sich die vollständige Verseifung. Eine passende Concentration scheint mir hat die Seife und in Consistenz der käuflichen Schmierseife ähnlich wird dieselbe, wenn man sie !auf $300 \%$ des angewandten Rüböls eindampft, in dem Falle enthält dieselbe in $100 \mathrm{Th}$. die Fettsäure aus 33,3 Th. Oel, und da sich der Werth einer Seife nach der Menge der darin enthaltenen verseiften Fettsäure abschätzt, so würden 2 Th. derselben annähernd gleichwerthig sein 1 Th. spanischer Seife, welche jetzt zur Bereitung von Seifenspiritus und flüssigem Opodeldoc dient.

Auf diese Weise bleibt die bisherige Art der Bereitung von Seifenspiritus und fluissigen Opodeldoc insofern unangetastet, als sich wieder eine nicht unerhebliche Menge freien Alkalis in diesen Präparaten befindet; erfült letzteres aber keinen medicinischen Zweck und findet die mit Wasser bereitete Kaliseife in Substanz keine andere Verwendung, als zur Lösung in Alkohol, so lässt sich die Bereitung von Seifenspiritus und fliussigem Opodeldoc dahin modificiren, dass man $100 \mathrm{Th}$. Rüböl mit $15 \mathrm{Th}$. KO in alkoholisoher Lösung zunächst bei $100^{\circ} \mathrm{C}$. mischt; die vollständige Bindung der Fettsäure vollzieht sich nach Zusatz der vorgeschriebenen Menge Alkohols in etwa derselben Zeit und bei derselben Temp., welche man jetzt gebraucht, um spanische Seife zu lösen. Bei Bereitung des Seifenspiritus empfiehlt es sich, das Wasser nach zuvor geschehener Lösung des Oeles zuzu- 
setzien, da dasselbe die vollständige Verseifung befördert and das Verdampfen des Alkohols vermindert. $100 \mathrm{Th}$. Rüböl entsprechen, wie oben angenommen, $300 \mathrm{Th}$. Kaliseife in Substanz oder 150 Th. spanischer Seife. Diese Methode der Darstellung von Seifenspiritus und flüssigem Opodeldoc ist insofern der Anwendung von Kaliseife in Substanz rorzuziehen, als man nicht nöthig hat, den Alkohol zu verjagen, um denselben nachträglich wieder zuzusetzen. Bei Anwendung von $15 \mathrm{Th}$. KO auf $100 \mathrm{Th}$. Oel hat man dennoch ein wenig freien Alkalis in der alkoholischen Lösung, doch möchte ich nicht rathen, erbeblich weniger KO anzuwenden, da man sonst Gefahr läuft, unverseiftes $\mathrm{Oel}$ in Lösung zu behalten, welches eich in der Kälte durch eine Trübung zu erkennen giebt.

Diejenigen Fachgenossen, welche an maassgebender Stelle die Einfuhrung der Kaliseife zur Bereitung von Seifenspiritus und fiüssigem Opodeldoc in die nächste Ausgabe der deutschen Pharmacopoë befürworten können, mögen meine Angaben prüfen und vielleicht verbessern.

Burgdorf im Juli 1872.

\section{Die Antiseptica.}

Von Dr. Heinrich Böhnke-Reich.

Antiseptica oder fäulnisswidrige Mittel sind solche, deren man sich bedient, um organieche Stoffe vor Fäulniss zu schützen. Ihr $\mathrm{Zweck}$ ist, Fäulniss entweder aufzuheben and zu unterbrechen oder gar nicht eintreten zu lassen. Ihre Zahl und die Art ihrer Anwendung ist eine so grosse und vielfältige, dass hier nur der wichtigsten Erwähnung geschehen soll, wobei wir Desinfection und Desinfectionsmittel nicht s peciell behandeln.

Es ist eine Erfahrungssache, dass besonders Feuchtigkeit verbunden mit $W$ ärme, namentlich Temperaturen von 12 bịs 